\title{
APLICAÇÃO DA TÉCNICA SR-TXRF PARA AVALIAÇÃO DO MECANISMO DE SORÇÃO DO METAL PESADO CD(II) PELA BIOMASSA EGERIA DENSA INATIVA
}

\author{
G.H.F. SANTOS ${ }^{1}$, A.N. MÓDENES ${ }^{2}$, F.R. ESPINOZA-QUIÑNONES ${ }^{2}$, A.R. TASCHIN ${ }^{2}$ e M.A.S.S. \\ RAVAGNANI ${ }^{1}$ \\ ${ }^{1}$ Universidade Estadual de Maringá, Departamento de Engenharia Química; \\ ${ }^{2}$ Universidade Estadual do Oeste do Paraná, Departamento de Engenharia Química \\ E-mail para contato: ghfidelis@ hotmail.com
}

\begin{abstract}
RESUMO - Neste trabalho foi realizado o estudo do mecanismo de biossorção do íon metálico cádmio utilizando como biossorvente a macrófita aquática Egeria densa. Experimentos em batelada foram conduzidos com temperatura e velocidade de agitação controlados por um período de 2 horas utilizando uma mistura de $300 \mathrm{mg}$ do biossorvente e $50 \mathrm{~mL}$ de solução metálica com concentração inicial de $224,8 \mathrm{mg} \mathrm{L}^{-1}$ (4 mequiv. $\mathrm{L}^{-1}$ ) e pH 5. A análise da concentração inicial e residual dos metais presentes em solução aquosa e no biossorvente foram realizadas pela técnica SR-TXRF (Fluorescência de Raio-X por Reflexão Total emitida por Radiação Síncrotron). A partir do balanço de massa entre os principais elementos envolvidos no processo de sorção, identificou-se que o principal mecanismo responsável pela remoção dos íons cádmio pela macrófita $E$. densa foi o processo de troca iônica.
\end{abstract}

\section{INTRODUÇÃO}

A contaminação do ambiente aquático por poluentes inorgânicos em baixas concentrações aumenta com o desenvolvimento industrial e o crescimento populacional. Os metais pesados são elementos que por possuírem alta solubilidade e mobilidade, tendem a acumular-se na cadeia alimentar podendo trazer sérios impactos à saúde do homem (Borba et al., 2008). Entre os metais pesados, o cádmio é encontrado em efluentes de indústrias envolvidas com chapeação metálica, ligas metalúrgicas, mineração, cerâmica, produção de baterias de níquel-cádmio, entre outras atividades (Chen et al., 2012). A bioacumulação deste metal no organismo humano pode causar diversas doenças como hipertensão arterial, enfisema pulmonar, anemia, câncer, disfunções renais, osteoporose, entre outras (Ding et al., 2012; Saif et al., 2012).

O tratamento de resíduos contaminados com metais pesados pode ser realizado por diversos métodos convencionais, como precipitação química, ultrafiltração com membranas, processos eletroquímicos, osmose reversa e processos de sorção como adsorção, troca iônica e biossorção (Fu e Wang, 2011). Dentre estas técnicas, a biossorção apresenta alta eficiência em reduzir a concentração de metais pesados em resíduos com um custo inferior a outros adsorventes mais comuns, como o 


\section{9 a 22 de outubro de 2014 \\ Florianópolis/SC}

carvão ativado (Lesmana et al., 2009).

Por este motivo, diferentes tipos de biomassa são estudados como biossorvente alternativo na remoção de metais pesados, como macroalgas, casca de laranja, plantas aquáticas, entre outros (Espinoza-Quiñones et al., 2009; Fu e Wang, 2011; Módenes et al., 2013). Devido a complexidade estrutural e os diversos grupos funcionais distribuídos na superfície dos biomateriais, diferentes mecanismos de transferência de massa podem estar envolvidos na biossorção, como troca iônica, adsorção, quelação, complexação e microprecipitação (Davis et al., 2003; Espinoza-Quiñones et al., 2013). A identificação destes mecanismos é de fundamental importância para a aplicação desta técnica a nível industrial, pois de acordo com as biomassas utilizadas, sua origem e seu processamento, um dos mecanismos podem ser predominantes. Este conhecimento possibilitará condições para aperfeiçoar o processo a nível molecular, desenvolver um processo economicamente mais viável e utilizar biossorventes mais baratos e eficientes (Farooq et al., 2010).

Logo, este trabalho tem por objetivo estudar os mecanismos envolvidos na remoção do íond cádmio pela macrófita $E$. densa inativa. Os elementos presentes em solução aquosa e no biossorvente serão quantificados pela técnica analítica SR-TXRF. A partir do balanço de massa em cada elemento detectado, será identificado o mecanismo envolvido na biossorção.

\section{MATERIAIS E MÉTODOS}

\subsection{Biossorvente e Solução Metálica}

A macrófita aquática $E$. densa foi coletada e preparada como descrito em trabalhos anteriores (Espinoza-Quiñones et al., 2009; Pietrobelli et al., 2009; Módenes et al., 2012). As soluções utilizadas nos experimentos foram preparadas com reagentes químicos analíticos com alto grau de pureza e água destilada/deionizada. As soluções metálicas de cádmio foram preparadas diluindo os sais $\mathrm{CdCl}_{2} \cdot 2 \mathrm{H}_{2} \mathrm{O}$ em água destilada/deionizada, e ajustando o $\mathrm{pH}$ em 5 . A concentração inicial das soluções de cádmio foram de 4 mequiv. $\mathrm{L}^{-1}\left(224,8 \mathrm{mg} \mathrm{L}^{-1}\right)$. Soluções de $\mathrm{HCl}(1 \mathrm{M})$ e $\mathrm{NaOH}(1 \mathrm{M})$ foram utilizadas para ajuste de $\mathrm{pH}$.

\subsection{Análise de $\mathrm{pH}_{\mathrm{pcz}}$}

Com base no modelo de complexação de superfície e na metodologia de titulação ácido-base, foi determiando o $\mathrm{pH}$ do ponto de carga zero $\left(\mathrm{pH}_{\mathrm{pcz}}\right)$ para a biomassa $E$. densa (Borba et al., 2010). Após adição de $100 \mathrm{~mL}$ de $\mathrm{NaNO}_{3}\left(0,1 \mathrm{~mol} \mathrm{~L}{ }^{-1}\right)$ em $5 \mathrm{~g}$ de biossorvente, soluções de $\mathrm{HNO}_{3}(0,1 \mathrm{~mol}$ $\left.\mathrm{L}^{-1}\right)$ e $\mathrm{NaOH}\left(0,1 \mathrm{~mol} \mathrm{~L}^{-1}\right)$ foram tituladas, sendo registado as quantidades de ácido e base necessárias para se atingir cada valor de $\mathrm{pH}$ ao longo de uma ampla faixa de $\mathrm{pH}$.

\subsection{Ensaios de biossorção de cádmio}

Uma mistura de $300 \mathrm{mg}$ de biossorvente e $50 \mathrm{~mL}$ de solução de cádmio $\left(224,8 \mathrm{mg} \mathrm{L} \mathrm{L}^{-1}\right)$ foram agitadas em uma incubadora refrigerada a temperatura controlada de $30{ }^{\circ} \mathrm{C}$ durante 2 horas (Pietrobelli et al., 2009; Módenes et al., 2012). Os experimentos de biossorção foram realizados em 


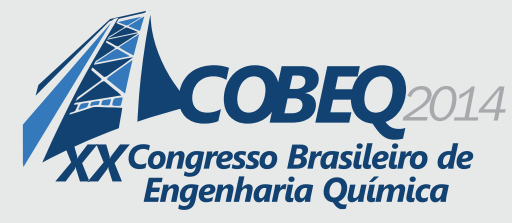

19 a 22 de outubro de 2014
Florianópolis/SC

triplicata. Após o período de agitação, as soluções foram filtradas em membrana nitrato de celulose com microporos de $0,45 \mu \mathrm{m}$ utilizando um aparato de filtração e bomba de vácuo. $\mathrm{O}$ biossorvente filtrado foi seco em estufa a $40{ }^{\circ} \mathrm{C}$ para posteriormente ser analisado. As análises das amostras líquidas e sólidas foram realizadas em quintoplicata pela técnica SR-TXRF.

\subsection{Análises SR-TXRF}

A macrófita seca foi digerida misturando-se $500 \mathrm{mg}$ da biomassa com $5 \mathrm{~mL}$ de ácido nítrico $65 \%$ e $0,5 \mathrm{~mL}$ de peróxido de hidrogênio $35 \%$. A digestão foi realizada em bloco digestor a $150{ }^{\circ} \mathrm{C}$ por 8 horas (Espinoza-Quiñones et al., 2009). Para uniformizar o resíduo da digestão, aferiu-se este a $10 \mathrm{~mL}$ com água ultra pura Mili-Q. Adicionou-se a uma alíquota de $900 \mu \mathrm{L}$ de amostra digerida, 100 $\mu \mathrm{L}$ de solução padrão de Gálio a $1000 \mathrm{mg} \mathrm{L}^{-1}$, resultando em uma concentração de $100 \mathrm{mg} \mathrm{L}^{-1}$ de Ga. Para as amostras líquidas, também foi misturado uma alíquota $900 \mu \mathrm{L}$ de amostra com $100 \mu \mathrm{L}$ da solução padrão de $\mathrm{Ga} 1000 \mathrm{mg} \mathrm{L}^{-1}$. Em seguida pipetou-se $5 \mu \mathrm{L}$ das amostras com $\mathrm{Ga}$ em refletores retangulares de acrílico. Os refletores-amostra foram secos em capela laminar.

Cada refletor-amostra foi irradiado por um período de 100 segundos sob reflexão total por um feixe policromático de raios-X, na faixa de 2 a $20 \mathrm{keV}$, extraído da fonte de Luz Síncrotron de 2,5 $\mathrm{GeV}$. Os raios-X foram detectados utilizando-se um detector $\mathrm{Si}(\mathrm{Li})$, com resolução de $165 \mathrm{eV}$ na linha espectral de Mn-Ka, e um colimador de tântalo. Os sinais do detector foram obtidos por uma eletrônica padrão para espectrometria de raios-X (Espinoza-Quiñones et al., 2010). De cada refletoramostra foi gerado um espectro SR-TXRF, sendo as intensidades de pico das linhas K calculadas pelo ajuste dos espectros com o programa AXIL (3.6 version).

Amostras de materiais referenciados, como "água fluvial" certificada pelo National Research Council Canadá, designado por NRC-CNRC/SLRS-4 (RIVERINE WATER) e folhas de tomate certificadas pelo National Institute for Science and Technology (NIST) designadas por SRM/RM 1573 a, foram irradiadas para garantir a confiabilidade dos resultados obtidos para as amostras líquidas e sólidas, respectivamente. A análise SR-TXRF para estes materiais seguiu o mesmo procedimento utilizado no preparo das amostras líquidas e sólidas.

\subsection{Determinação da concentração elementar}

A partir da intensidade fluorescente das linhas de raios- $\mathrm{X} \mathrm{K}$ de cada elemento $\left(\mathrm{N}_{\mathrm{i}}\right)$ relativo a intensidade fluorescente das linhas de raios-X K do padrão interno $\mathrm{Ga}\left(\mathrm{N}_{\mathrm{Ga}}\right)$, da concentração de gálio na amostra $\left(\mathrm{C}_{\mathrm{Ga}}\right)$ e da curva de sensibilidade elementar $\left(\mathrm{S}_{\mathrm{i}}\right)$ do espectrômetro SR-TXRF, a concentração elementar na fase líquida $\left(C_{i}\right)$ foi calculada pela Equação (1).

$$
C_{i}=\frac{N_{i}}{N_{G a}} \frac{C_{G a}}{S_{i}}
$$

Para determinação das concentrações dos elementos presentes na fase sólida $\left(\mathrm{C}_{\mathrm{FS}}, \mathrm{i}\right)$ foi utilizado a Equação (2), onde converte-se a concentração da fase líquida para fase sólida. 


$$
C_{F S, i}=C_{i} \frac{V}{M}
$$

Em que $\mathrm{M}$ é a massa de biomassa digerida e $\mathrm{V}$ é o volume aferido com água Mili-Q. Para obtenção da curva de sensibilidade elementar da técnica SR-TXRF para a linha espectral Ka, foram preparadas soluções padrão multielementares com 20 elementos diferentes.

\section{RESULTADOS E DISCUSSÕES}

\subsection{Teste Preliminar}

Um conjunto de grupos funcionais, tais como hidroxil, carboxil e fosforil são normalmente encontrados na superfície da biomassa (Ahluwalia e Goyal, 2007). A superfície de um biossorvente pode estar carregada positiva ou negativamente, dependendo do valor do $\mathrm{pH}$ da solução e da estrutura da biomassa. No entanto, dependendo do pH da solução, as cargas da superfície de um biomaterial podem se encontrar neutralizadas, sendo denominado por $\mathrm{pH}$ do ponto de carga zero $\left(\mathrm{pH}_{\mathrm{pcz}}\right)$. De acordo com o $\mathrm{pH}_{\mathrm{pcz}}$ da macrófita $E$. densa, ilustrado na Figura 1, a distribuição das cargas na superfície deste biossorvente foi entre 5,0-6,5. De acordo com o diagrama de especiação metálica obtido pelo software Hydra (Borba et al., 2010), as formas iônicas, $\mathrm{Cd}^{2+}$ e $\mathrm{CdCl}^{+}$, estão presentes na solução até pH 8 na razão 3:1, enquanto que precipitados de $\mathrm{CdOH}_{2(\text { (r) })}$ se formam com pH acima de 8 . A partir dos resultados do $\mathrm{pH}_{\mathrm{pcz}}$ e da especiação metálica, e para evitar a formação de precipitados, os experimentos foram conduzidos com $\mathrm{pH} 5$.

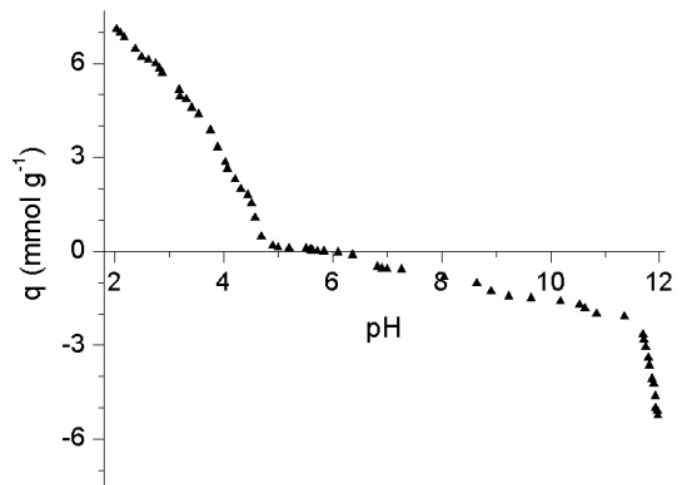

Figura 1 - Distribuição das cargas na superfície da macrófita aquática Egeria densa inativa.

\subsection{Resultados SR-TXRF}

A partir das concentrações elementares das soluções padrões multielementares com 20 elementos diferentes, os valores da sensibilidade experimental do espectrômetro SR-TXRF para a linha de raio- $\mathrm{X} \mathrm{K \alpha}$ relativa ao gálio foram obtidas e ajustadas aplicando uma função do tipo exponencial dependente do número atômico $(\mathrm{Z})$. A curva de sensibilidade para a linha espectral Ka é descrita pela Equação (3). Utilizando o software AXIL, as áreas dos picos para as linhas Ka foram 
determinadas, com objetivo de quantificar as concentrações dos elementos detectados nas fases líquidas e sólidas.

$$
S_{K}=\exp \left[-26,41+1,615 z-0,02461 z^{2}\right]\left(r^{2}=0,9996\right)
$$

Os resultados obtidos para as amostras dos padrões certificados de "água fluvial" e folhas de tomate para os principais elementos detectados nos experimentos de biossorção do cádmio são apresentados na Tabela 1. Estes resultados mostraram boa porcentagem de recuperação na concentração de cada elemento identificado, com um desvio padrão abaixo de $5 \%$.

Tabela 1 - Comparação entre os valores de concentração elementar certificados pelos padrões, NRC-CNRC - material de referência de água de rio SLRS-4 e NIST - material de referência de folha de tomate 1573a, e os valores medidos pela técnica SR-TXRF

\begin{tabular}{lcccccc}
\hline \multirow{2}{*}{ Elementos } & \multicolumn{2}{c}{ Material de referência de água de rio SLRS-4 } & \multicolumn{3}{c}{ Material de referência de folha de tomate 1573a } \\
\cline { 2 - 7 } & $\begin{array}{c}\text { Valor } \\
\text { Certificado } \\
\left(\mathrm{mg} \mathrm{L}^{-1}\right)\end{array}$ & $\begin{array}{c}\text { Valor Medido } \\
\left(\mathrm{mg} \mathrm{L}^{-1}\right)\end{array}$ & $\begin{array}{c}\% \\
\text { recuperação }\end{array}$ & $\begin{array}{c}\text { Valor } \\
\text { Certificado } \\
\left(\mathrm{mg} \mathrm{g}^{-1}\right)\end{array}$ & $\begin{array}{c}\text { Valor Medido } \\
\left(\mathrm{mg} \mathrm{g}^{-1}\right)\end{array}$ & $\%$ recuperação \\
\hline Cálcio & $6,2 \pm 0,2$ & $6,0 \pm 0,3$ & 96,8 & $50,5 \pm 0,9$ & $48,7 \pm 3,9$ & 96,4 \\
Potássio & $0,68 \pm 0,02$ & $0,67 \pm 0,02$ & 98,5 & $27,0 \pm 0,5$ & $25,9 \pm 2,1$ & 95,9 \\
Ferro & $103 \pm 5$ & $106 \pm 6$ & 102,9 & $0,368 \pm 0,007$ & $0,386 \pm 0,031$ & 104,9 \\
Manganês & $3,37 \pm 0,18$ & $3,22 \pm 0,20$ & 95,5 & $0,246 \pm 0,008$ & $0,252 \pm 0,020$ & 102,4 \\
\hline
\end{tabular}

Na Tabela 2 são apresentados os valores de concentração de cada elemento na fase aquosa e na biomassa antes e após o processo de biossorção. Nos experimentos de remoção do cádmio, os principais elementos identificados e quantificados pela técnica SR-TXRF, nas amostras líquidas e sólidas foram $\mathrm{Cd}, \mathrm{Ca}, \mathrm{K}, \mathrm{Fe}, \mathrm{Mn}, \mathrm{S}$ e Ti. O Na é um elemento que também está presente no processo de biossorção, porém não foi possível detectá-lo por meio da técnica SR-TXRF. Portanto, as concentrações de sódio foram determinadas pela técnica de espectrofotometria de chama.

Tabela 2 - Valores das concentrações médias de cada elemento $(n=5)$ nas soluções aquosas (fase líquda) e no biossorvente (fase sólida), antes e após o processo de remoção dos íons cádmio

\begin{tabular}{|c|c|c|c|c|}
\hline \multirow[t]{2}{*}{ Elementos } & \multicolumn{2}{|c|}{$\begin{array}{c}\text { Concentração dos elementos na fase } \\
\text { líquida }\left(\mathrm{mg} \mathrm{L}^{-1}\right)\end{array}$} & \multicolumn{2}{|c|}{$\begin{array}{l}\text { Concentração dos elementos na fase } \\
\text { sólida }\left(\mathrm{mg} \mathrm{g}^{-1}\right)\end{array}$} \\
\hline & Antes & Depois & Antes & Depois \\
\hline $\mathrm{Cd}$ & $229,0 \pm 6,8$ & $72,4 \pm 1,9$ & $\leq 0,11$ & $26,5 \pm 1,3$ \\
\hline $\mathrm{Ca}$ & $8,7 \pm 0,7$ & $39,4 \pm 2,0$ & $23,1 \pm 1,2$ & $17,9 \pm 0,9$ \\
\hline $\mathrm{K}$ & $10,1 \pm 0,2$ & $20,9 \pm 1,4$ & $1,9 \pm 0,3$ & $0,4 \pm 0,1$ \\
\hline $\mathrm{Fe}$ & $0,47 \pm 0,07$ & $0,50 \pm 0,06$ & $9,4 \pm 0,3$ & $9,5 \pm 0,3$ \\
\hline Mn & $\leq 0,007$ & $11,5 \pm 0,9$ & $4,3 \pm 0,4$ & $2,25 \pm 0,11$ \\
\hline S & $\leq 0,2$ & $\leq 0,2$ & $0,57 \pm 0,03$ & $0,56 \pm 0,03$ \\
\hline $\mathrm{Ti}$ & $\leq 0,02$ & $\leq 0,02$ & $0,20 \pm 0,03$ & $0,23 \pm 0,03$ \\
\hline$(\mathrm{Na})^{*}$ & $2,0 \pm 0,2$ & $5,3 \pm 0,4$ & $0,74 \pm 0,08$ & $0,25 \pm 0,02$ \\
\hline
\end{tabular}

*Concentração determinada pela espectrofotometria de chamas. 

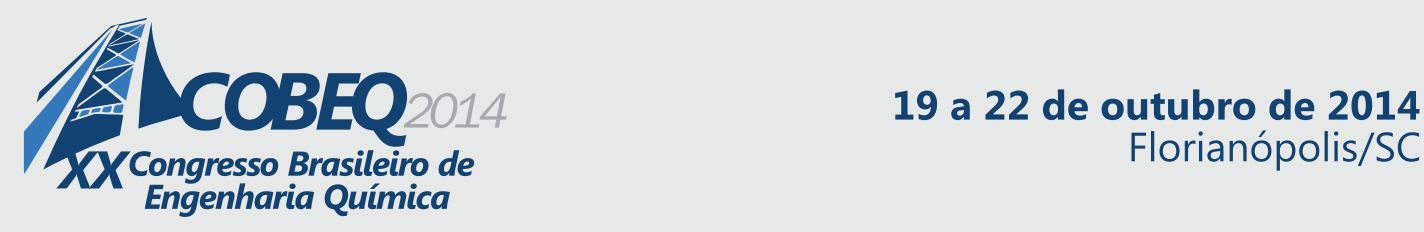

\section{Mecanismo de biossorção do cádmio}

Nos experimentos de biossorção do cádmio pela macrófita $E$. densa, observou-se que as concentrações de $\mathrm{Ca}, \mathrm{K}, \mathrm{Fe}, \mathrm{Mn}, \mathrm{S}$, Ti e Na na solução inicial são relativamente pequenas. Porém, após o contato da solução de cádmio com a biomassa inativa, as concentrações de alguns cátions como $\mathrm{Ca}, \mathrm{K}, \mathrm{Mn}$ e $\mathrm{Na}$ sofreram um aumento na concentração na fase líquida, enquanto que a concentração do $\mathrm{Cd}$ nesta fase diminuiu. Esta alteração coincidiu com a redução na concentração de $\mathrm{Ca}, \mathrm{K}, \mathrm{Na}$ e Mn na fase sólida, bem como com o aumento da concentração de Cd nesta mesma fase, que mostra que o metal se aderiu à biomassa (Tabela 2). As concentrações de Fe, $\mathrm{S}$ e Ti apresentaram variações pouco significantes.

Para uma análise quantitativa adequada na busca de maior evidência sobre o mecanismo envolvido no processo de remoção de $\mathrm{Cd}$, as concentrações elementares $\left(\mathrm{C}_{\mathrm{i}}\right.$, em mg $\mathrm{L}^{-1}$ ou mg g $\left.\mathrm{m}^{-1}\right)$ foram convertidas para equivalente molar $\left(\mathrm{m}_{\mathrm{i}}\right.$, em mequiv) de acordo com a Equação (4), onde o estado iônico de cada elemento $\left(\mathrm{q}_{\mathrm{i}}\right)$, a massa atômica $\left(\mathrm{A}_{\mathrm{i}}\right.$, em $\left.\mathrm{mg} \mathrm{mol}^{-1}\right)$, o volume de solução e a massa de biossorvente $(\delta$, L ou g) utilizados nos experimentos foram considerados (Tabela 3 ).

$$
m_{i}=\frac{C_{i} \delta q_{i}}{A_{i}}
$$

Tabela 3 - Balanço molar equivalente dos principais elementos detectados nas fases líquidas e sólidas antes e após o processo de remoção do cádmio

\begin{tabular}{ccccccc}
\hline \multirow{2}{*}{ Cátions } & \multicolumn{3}{c}{ Fase Líquida } & \multicolumn{3}{c}{ Fase Sólida } \\
\cline { 2 - 6 } & mequiv $_{\text {antes }}$ & mequiv $_{\text {depois }}$ & $\Delta$ (mequiv) & mequiv $_{\text {antes }}$ & mequiv $_{\text {depois }}$ & $\Delta($ mequiv $)$ \\
\hline $\mathrm{Cd}^{2+} / \mathrm{CdCl}^{+}$ & $4,14 \pm 0,15$ & $1,31 \pm 0,04$ & $-2,83 \pm 0,09$ & $\leq 0,015$ & $2,87 \pm 0,14$ & $+2,87 \pm 0,14$ \\
$\mathrm{Ca}^{2+}$ & $0,52 \pm 0,04$ & $2,36 \pm 0,12$ & $+1,84 \pm 0,07$ & $8,30 \pm 0,41$ & $6,42 \pm 0,32$ & $-1,88 \pm 0,09$ \\
$\mathrm{~K}^{+}$ & $0,31 \pm 0,02$ & $0,64 \pm 0,04$ & $+0,33 \pm 0,02$ & $0,35 \pm 0,06$ & $0,07 \pm 0,02$ & $-0,28 \pm 0,04$ \\
$\mathrm{Fe}^{2+}$ & $0,020 \pm 0,003$ & $0,021 \pm 0,003$ & $\leq 0,003$ & $2,42 \pm 0,08$ & $2,46 \pm 0,08$ & $\leq 0,05$ \\
$\mathrm{Mn}^{2+}$ & $\leq 0,001$ & $0,50 \pm 0,04$ & $+0,50 \pm 0,04$ & $1,13 \pm 0,10$ & $0,59 \pm 0,04$ & $-0,54 \pm 0,04$ \\
$\mathrm{~S}^{6+}$ & $\leq 0,04$ & $\leq 0,04$ & $\leq 0,04$ & $0,77 \pm 0,04$ & $0,76 \pm 0,04$ & $\leq 0,04$ \\
$\mathrm{Ti}^{4+}$ & $\leq 0,002$ & $\leq 0,002$ & $\leq 0,002$ & $0,12 \pm 0,02$ & $0,14 \pm 0,01$ & $\leq 0,02$ \\
$\left(\mathrm{Na}^{+}\right)^{*}$ & $0,10 \pm 0,02$ & $0,28 \pm 0,02$ & $+0,18 \pm 0,02$ & $0,23 \pm 0,03$ & $0,08 \pm 0,01$ & $-0,15 \pm 0,01$ \\
$\Sigma\left(\right.$ mequiv $_{\mathrm{i}}$ & $5,09 \pm 0,05$ & $5,11 \pm 0,05$ & $\leq 0,06$ & $13,32 \pm 0,07$ & $13,39 \pm 0,05$ & $\leq 0,08$ \\
\hline
\end{tabular}

*Concentração determinada pela espectrofotometria de chamas.

Pela análise SR-TXRF, observou-se que os cátions $\mathrm{Ca}^{2+}, \mathrm{K}^{+}, \mathrm{Mn}^{2+}$ e $\mathrm{Na}^{+}$(Tabela 3) foram liberados pela superfície da biomassa quando em contato com a solução de $\mathrm{Cd}$, o que evidencia que estes cátions estão envolvidos na remoção do metal. Por outro lado, a variação nas concentrações em mequiv de Fe, S e Ti na fase liquida e sólida foram insignificantes e negligenciáveis, em comparação com o desvio padrão. Pela análise das variações positivas e negativas $(\Delta \pm)$ em equivalente na fase líquida dos cátions envolvidos no processo, a soma das variações de $\mathrm{Ca}^{2+}(+1,84 \pm 0,07), \mathrm{K}^{+}$ $(+0,33 \pm 0,02), \mathrm{Mn}^{2+}(+0,50 \pm 0,04)$ e $\mathrm{Na}^{+}(+0,18 \pm 0,02)$ liberado pela biomassa foi de $(+2,85 \pm 0,06)$, que é a mesma variação de $\mathrm{Cd}^{2+} / \mathrm{Cl}^{+}(-2,83 \pm 0,09)$ removidos pelo biossorvente, dentro de um desvio 


\section{9 a 22 de outubro de 2014 \\ Florianópolis/SC}

padrão. Este mesmo resultado foi obtido analisando a soma das variações em equivalente dos cátions com o metal pesado na fase sólida.

Neste trabalho, a curva de $\mathrm{pH}_{\mathrm{pcz}}$ para a $E$. densa mostrou uma forte evidência da existência de diferentes tipos de grupos funcionais, na faixa de $\mathrm{pH} 3$ a 11, que poderiam contribuir com a captura do íons $\mathrm{Cd}^{2+} / \mathrm{CdCl}^{+}$, os quais reagem com os cátions ligados à superfície, liberando $\mathrm{Ca}^{2+}, \mathrm{K}^{+}, \mathrm{Mn}^{2+} \mathrm{e}$ $\mathrm{Na}^{+}$, como observado nos resultados mostrados acima (Tabela 3). A taxa de remoção de metais pesados pela macrófita $E$. densa aumenta com o aumento do pH entre 3 e 5 como resultado da protonação dos sítios ativos em valores de $\mathrm{pH}$ mais baixos (Pietrobelli et al., 2009).

De acordo com as evidências experimentais na remoção de Cd pela macrófita $E$. densa em $\mathrm{pH}$ 5, a quantidade específica deste metal removida em mequiv e a presença de outros cátions liberados pela superfície, o mecanismo envolvido na remoção de cádmio é o processo de troca iônica na superfície do biossorvente.

\section{CONCLUSÕES}

A técnica SR-TXRF é uma excelente ferramenta analítica na medição da concentração de elementos presentes em amostras sólidas e líquidas, que permitiu a determinação da concentração dos principais elementos envolvidos no processo de biossorção de $\mathrm{Cd}$ pela macrófita E. densa. A recuperação obtida na análise de padrões certificados validou os resultados encontrados para a remoção dos íons cádmio utilizando a técnica SR-TXRF. Avaliando as variações significantes em equivalente para cada cátion analisado antes e após o processo de remoção do cádmio, $\mathrm{Ca}^{2+}, \mathrm{K}^{+}, \mathrm{Mn}^{2+}$ e $\mathrm{Na}^{+}$foram identificados como sendo os principais elementos envolvidos neste processo. Estes cátions foram liberados da superfície do biossorvente para a solução aquosa, enquanto que os íons $\mathrm{Cd}^{2+} / \mathrm{CdCl}^{+}$foram aderidos a superfície do biossorvente em $\mathrm{pH}$ 5. Portanto, o processo de troca iônica foi confirmado como principal mecanismo responsável pela remoção dos íons Cd pela macrófita aquática Egeria densa inativa.

\section{REFERÊNCIAS BIBLIOGRÁFICAS}

AHLUWALIA, S.S.; GOYAL, D. Review: Microbial and plant derived biomass for removal of heavy metals from wastewater. Bioresour. Technol., v. 98, p. 2243-2257, 2007.

BORBA, C.E.; SILVA, E.A.; FAGUNDES-KLEN, M.R.; KROUMOV, A.D.; GUIRARDELLO, R. Prediction of the copper (II) ions dynamic removal from a medium by using mathematical models with analytical solution. J. Hazard. Mater., v. 152, p. 366-372, 2008.

BORBA, C.E.; SILVA, E.A.; SPOHR, S.; SANTOS, G.H.F.; GUIRARDELLO, R. Ion exchange equi-librium prediction for the system $\mathrm{Cu} 2+-\mathrm{Zn} 2+-\mathrm{Na}+$, J. Chem.. Eng. Data, v. 55, p. 1333$1341,2010$.

DAVIS, T.A.; VOLESKY, B.; MUCCI, A. A review of the biochemistry of heavy metal biosorption by brown algae. Water Res., v. 37, p. 4311.4330, 2003. 
DING, Y.; JING, D.; GONG, H.; ZHOU, L.; YANG, X. Biosorption of aquatic cadmium(II) by unmodified rice straw. Bioresour. Technol., v. 114, p. 20-25, 2012.

ESPINOZA-QUIÑONES, F.R.; RIZZUTTO, M.A.; ADDED, N.; TABACNIKS, M.H.; MÓDENES, A.N.; PALÁCIO, S.M.; SILVA, E.A.; ROSSI, F.L.; MARTIN, N.; SZYMANSKI, N. PIXE analysis of chromium phytoaccumulation by the aquatic macrophytes Eicchornia crassipes, Nucl. Instrum. Methods Phys. Res., Sect B, v. 267, p. 1153-1157, 2009.

ESPINOZA-QUIÑNONES, F.R.; PALÁCIO, S.M.; MÓDENES, A.N.; SZYMANSKI, N.; ZACARKIM, C.E.; ZENATTI, D.C.; FORNARI, M.M.T.; RIZZUTTO, M.A.; TABACNIKS, M.H.; ADDED, N.; KROUMOV, A.D. Water quality assessment of Toledo River and determination of metal concentrations by using SR-TXRF technique. J. Radioanal. Nucl. Chem., v. 283, p. 465-470, 2010.

ESPINOZA-QUIÑONES, F.R.; MÓDENES, A.N.; SANTOS, G.H.F.; BORBA, C.E.; RIZZUTTO, M.A.; RAVAGNANI, M.A.S.S. Thin and thick target PIXE analyses to assess the mechanism of $\mathrm{Cu}^{2+}$ removalby Egeria densa. Appl. Radiat. Isto., v. 82, p. 1-6, 2013.

FAROOQ, U.; KOZINSKI, J.A.; KHAN, M.A.; ATHAR, M. Biosorption of heavy metal ions using wheat based biosorbents: a review. Bioresour. Technol., v. 101, p. 5043-5053, 2010.

FU, F.; WANG, Q. Removal of heavy metal ions from wastewaters: a review. J. Environ. Manage., v. 92, p. 407-418, 2011.

LESMANA, S.O.; FEBRIANA, N.; SOETAREDJO, F.E.; SUNARSO, J.; ISMADJI, S. Studies on potencial applications of biomass for the separation of heavy metals from water and wastewater. Biochem. Eng. J., v. 44, p. 19-41, 2009.

MÓDENES, A.N.; ESPINOZA-QUIÑNONES, F.R.; TRIGUEROS, D.E.G.; PIETROBELLI, J.M.T.A.; LAVARDA, F.L.; RAVAGNANI, M.A.S.S.; BERGAMASCO, R. Binary Adsorption of a $\mathrm{Zn}$ (II)-Cu(II) Mixture onto Egeria densa and Eichhornia crassipes: Kinetic and Equilibrium Data Modeling by PSO. Sep. Sci. Technol., v. 47, p. 875-885, 2012.

MÓDENES, A.N.; ESPINOZA-QUIÑONES, F.R.; SANTOS, G.H.F.; BORBA, C.E.; RIZZUTTO, M.A. Assessment of metal sorption mechanisms by aquatic macrophytes using PIXE analysis. J. Hazard. Mater., v. 261, p. 148-154, 2013.

PIETROBELLI, J.M.T.A.; MÓDENES, A.N.; FAGUNDES-KLEN, M.R.; ESPINOZA-QUIÑONES, F.R. Cadmium, copper and zinc biosorption study by non-living Egeria densa biomass. Water Air Soil Pollut., v. 202, p. 385-392, 2009.

SAIF, M.M.S.; KUMAR, N.S.; PRASAD, M.N.V. Binding of cadmium to Strychnos potatorum seed proteins in aqueous solution: Adsorption kinetics and relevance to water purification. Colloids Surf., B., v. 94, p. 73-79, 2012. 